\title{
SOZIO-ÖKONOMISCHER WANDEL UND VERFASSUNGSREFORM IN CHILE 1925-1972
}

\author{
Von Dieter Nohlen
}

Unter den Ländern Lateinamerikas sticht Chile durch eine kontinuierliche Verfassungstradition und durch ein stabiles politisches System hervor, das erst jüngst durch die Programmatik und poltische Strategie der Allende-Regierung den hohen Grad an Legitimität und Konsensus der Bevölkerung einzubüßen scheint. Martin Needler räumte Chile den ersten Rang in "constitutional government" Lateinamerikas ein ${ }^{1}$. Der chilenischen Oberschicht war es bereits in der ersten Hälfte des 19. Jahrhunderts gelungen, auf der Grundlage der Verfassung von 1833 einen zentralistisch und autokratisch regierten Staat aufzubauen, in welchem von wenigen Ausnahmen abgesehen das Militär eine den Zivilisten nachgeordnete Rolle in der Politik spielte2.

Die Kontinuität des politischen Lebens in Chile drückt sich einerseits im minimalen Verfassungsverschleiß seit 1833 aus, andererseits in der Tradition der Parteien und des Parteiensystems. Die Verfassung von 1833, die präsidial strukturiert war, wurde während der Zeit ihrer Gültigkeit nur wenig geändert. Zentral war die durch die Ausbreitung des Wahlrechts bedingte periodische Reform der die Wahl der Verfassungsorgane betreffenden Artikel $^{3}$. Die langlebige 1833er Verfassung wurde 1925 durch eine neue Grundordnung abgelöst, weniger aus dem Bedürfnis heraus, das nach der Traditionsverfassung vorgesehene politische System zu verändern. Vielmehr fußte die neue Verfassung auf den Strukturprinzipien der alten und stellte nach einer 1891 beginnenden Periode, in welcher das Parlament ein verfassungspolitisches Übergewicht gegenüber dem Präsidenten besaß und ein dem klassischen französischen Parlamentarismus ähnliches parlamentarisches Regime praktiziert wurde ${ }^{4}$, das präsidentielle Regierungssystem mit eindeutiger Vorrangstellung des Präsidenten wieder her.

Ebenso bedeutsam wie die Dauerhaftigkeit der politischen Verfassung sind die starke historische Kontinuität der politischen Parteien in Chile trotz grundlegender Veränderung der sozio-ökonomischen und politischen Konfliktmuster und die unveränderte Vielparteienstruktur des chilenischen Parteiensystems $s^{5}$. Den traditionellen Parteien gelang es, neue soziale Schichten in das politische System zu integrieren und sie relativ rasch den für seine Stabilität wichtigen Konsensus über

1 Martin C. Needler, Political Development and Socio-Economic Development: The Case of Latin America, in: AmPSR, 62/1968, S. 891.

$2 \mathrm{Zu}$ den frühen Verfassungen siehe noch immer die klassische Studie von Ramon Briseño, Memoria histórico-crítica del Derecho Público Chileno desde 1810 hasta nuestros días, Santiago 1849; die Verfassungstexte Chiles sind am besten zugänglich in der zweibändigen Publikation von Luis Valencia Avaria, Anales de la República. Textos Constitucionales de Chile y Registro de los Ciudadanos, Santiago 1951.

3 Siehe dazu Fernando Campos Harriet, Historia constitucional de Chile, Santiago 1956, 4. Aufl. 1969; speziell zur Wahlrechtsentwicklung in verfassungsrechtlicher Perspektive: Luis Valdés Larrain, El sufragio, Santiago 1940. Eine detaillierte Darstellung von Wahlrecht und Wahlsystem enthält mein Beitrag "Chile ${ }^{\star}$ für das Handbuch "Die Wahl der Parlamente“, hrsg. von Dolf Sternberger, Bernhard Vogel mit Dieter Nohlen, Klaus Landfried, Band III, Wahlen und politischer Wandel in Amerika, von Dieter Nohlen und Rainer-Olaf Schultze, 1. Halbband, in Vorbereitung.

4 Zum Regierungstyp siehe Karl Loewenstein, Verfassungslehre, Tübingen 1959, S. 86-89, zum Parlamentarismus à la française in Chile Paul S. Reisch, Parliamentary Government in Chile, in: AmPSR, 3/1909, S. $507 \mathrm{ff}$

5 Die Vielparteienstruktur des chilenischen Parteiensystems geht auf das 19. Jahrhundert zurück. Die beiden großen Gruppierungen, Konservative und Liberale, waren in sich vielfach zergliedert. 1858 wurde die Radikale Partei, 1887 die Demokratische Partei (Vorläufer der Kommunistischen Partei) gegründet. Siehe dazu in mehr ideengeschichtlicher Perspektive Ricardo Donoso, Las ideas políticas en Chile, Mexico 1946, 2. Aufl. Santiago 1967 und Germán Urzúa Valenzuela, Los partidos políticos chilenos. Las fuerzas políticas, Santiago 1968. 
die Verfassung als legitime Ordnung des gesellschaftlichen Konflikts gewinnen zu lassen. Trotz Aufrechterhaltung von Manipulationen bei Wahlen bis in die fünfziger Jahre hinein haben die Wahlergebnisse stets die Anerkennung durch die Parteien gefunden. Der zumindest in unserem Jahrhundert formal offene Stromkreis der Macht hat zudem zu einer Lebendigkeit des politischen Prozesses in Chile geführt, die kaum in einem anderen Land anzutreffen ist. Politik oder Politisieren ist ein zentrales Lebenselement der Chilenen.

In den über zweieinhalb Jahrhunderten politischer Unabhängigkeit haben sich in Chile politische und soziale Institutionen herausgebildet, die mit denen der westlichen Demokratien durchaus vergleichbar sind. Die Diskrepanz liegt allerdings darin, daß Wirtschaftsstruktur und Produktionsniveau entweder unverändert geblieben oder den gesellschaftlichen Anforderungen nicht nachgekommen sind. Es erfolgte zwar eine Adaptation von Modernisierungselementen, doch die traditionalen Strukturen in Wirtschaft und Gesellschaft blieben aufrechterhalten. Diese Kluft zwischen politischer Entwicklung und sozio-ökonomischer Entwicklung konnte spätestens seit Beginn der sechziger Jahre nicht länger fortdauern ${ }^{6}$. Die Lage der sozial rückständigen Bevölkerungsteile und die ökonomische Stagnation begründeten neue Anforderungen an die politischen Parteien und an die politische Verfassung, deren Reform eine notwendige Voraussetzung des Strukturwandels in Wirtschaft und Gesellschaft wurde.

\section{Die chilenische Verfassung vom 18. September 1925}

Beratungen und Reformen der Verfassung von 1925 wurden stark von den Erfahrungen beeinflußt, die das Land mit der 1833er-Verfassung gemacht hatte ${ }^{7}$. So versuchte man, dem Parlament Rechte zu nehmen, die historische Grundlage dafür gewesen sind, daß an die Stelle des Präsidenten das Parlament in den Mittelpunkt des Regierungssystems gerückt ist.

Das chilenische Verfassungssystem ist gewaltenteilig strukturiert ${ }^{8}$. Der Präsident der Republik ist der Chef der Exekutive. Er beruft nach seinem Gutdünken die Minister, die Staatssekretäre, die Intendanten der Provinzen und die Gouverneure der Departements sowie die Bürgermeister der wichtigsten Städte (VfsArt. 72). Er ist der oberste Dienstherr der Streitkräfte, die er aber normalerweise nicht befehligt. Die obersten Militärs unterstehen seinem Vertrauen; er kann sie in den Ruhestand versetzen, doch bedürfen Neuernennungen der Zustimmung des Senats (VfsArt. 72,7). Der Kongreß hat im wesentlichen legislative Funktionen; er besteht aus Senat (heute 50 Mitglieder) und Abgeordnetenhaus (heute 150 Mitglieder), deren Mitgliederzahl sich nur geringfügig erhöht hat. Regierungsamt und Parlamentsmandat sind inkompatibel (VfsArt. 28). Präsident und Parlament teilen sich in das Recht der Gesetzesinitiative (VfsArt. 45), doch wurde dem Präsidenten im Bereich des Staatshaushalts eine dominierende Stellung eingeräumt. Die Kom-

6 Vgl. Aníbal Pinto, Desarrollo económico y relaciones sociales en Chile, in: Ders., Tres ensayos sobre Chile y América Latina, Buenos Aires 1971, S. 67-123, hier S. 67, $78 \mathrm{f}$.

$7 \mathrm{Zu}$ den Verfassungsberatungen s. Ministerio del Interior, Actas oficiales de las sesiones celebradas por la Comisión y Subcomisiones encargadas del estudio del Proyecto de Nueva Constitución Política de la República, Santiago 1925.

8 Eine mehr normative Analyse der Verfassung in ihren Grundzügen und Entwicklungstendenzen findet sich bei Wolfgang Prieur Koelling, Die Verfassung von Chile. Eine Anleitung zum Studium von Staatsrecht und Innerer Verwaltung in der Republik Chile, Frankfurt-Berlin $1964(=$ Die Staatsverfassungen der Welt in Einzelausgaben, Bd. 6). Die beste Studie des chilenischen Regierungssystems stammt von Federico G. Gil, The Political System of Chile, Boston 1967, erweiterte und korrigierte spanischsprachige Ausgabe El sistema político de Chile, Santiago 1969. 
petenz des Parlaments, Änderungen zu beschließen, wurde eng begrenzt und mit der Auflage versehen, bei Ausgabenerhöhungen zugleich die erforderlichen Dekkungsmöglichkeiten zu schaffen oder anzugeben (Vfs Art. 44,4). Die Beratungszeit des Haushaltsplangesetzes im Parlament wurde auf vier Monate begrenzt. In der Verfassungswirklichkeit verstärkte sich der Vorrang des Präsidenten in der Gesetzgebung durch die verfassungsrechtlich nicht vorgesehene, in den Beratungen abgelehnte Delegation der Gesetzgebungskompetenz des Kongresses an den Präsidenten in Form der Gesetzgebung per Dekret (Decretos con Fuerza de Ley, DFL) ${ }^{9}$. Die Gesetzgebung auf Initiative des Parlaments kann durch ein Veto des Präsidenten abgeblockt werden, das nur durch eine Zweidrittelmehrheit in beiden Kammern des Kongresses überwunden werden kann (VfsArt. 54). Das Gegengewicht des Parlaments darf jedoch nicht unterschätzt werden. Es hat sich zwar nicht so stark erwiesen, daß es erneut die Regierung vom Kongreß abhängig machen konnte. Doch war das Parlament vielfach in der Lage, die Reformgesetzgebung eines Präsidenten vollkommen zu blockieren. Eine Analyse der Verfassungsnormen muß hier die Vielparteienstruktur des chilenischen Parteiensystems mit berücksichtigen, das - legt man das Rechts-Links-Kontinuum zugrunde - drei etwa gleich starke Blöcke aufweist: die Rechte, die Mitte und die Linke ${ }^{10}$. Da die Präsidenten oft aus einem der Blöcke hervorgehen, verbinden sich die anderen beiden Blöcke in der Opposition gegen die Initiativen des Präsidenten. So wird verständlich, daß bereits die bloße Forderung nach Zustimmung des Kongresses zu den Gesetzesvorlagen des Präsidenten ein erhebliches Kontroll- und Obstruktionspotential des Parlaments darstellte, zumal ein Konflikt zwischen beiden Verfassungsorganen nicht durch Anrufung der Wählerschaft entschieden werden konnte ${ }^{11}$. Die politische Verantwortlichkeit der Minister kann der Kongreß durch Ministeranklagen, die vom Abgeordnetenhaus eingebracht und vom Senat entschieden werden (VfsArt. 39 und 42), verwirklichen ${ }^{12}$. Wahlberechtigt waren 1925 alle männlichen Chilenen über 21 Jahren, die lesen und schreiben konnten und in die Wahlregister eingetragen waren. Alle Mandatsträger wurden vom Volk nach gleichem, direktem und geheimem Wahlrecht gewählt, der Präsident auf sechs Jahre, wobei die Wiederwahl in direkter Folge ausgeschlossen ist, das Abgeordnetenhaus auf vier Jahre, der Senat auf acht Jahre bei Halberneuerung alle vier Jahre gleichzeitig mit der Wahl der zweiten Kammer (Vfs Art. 7, 38, 41 und 62). Das Wahlrecht wurde kontinuierlich auf ein heute allgemeines Wahlrecht ausgedehnt, worauf noch näher einzugehen ist; das Wahlsystem blieb indes unverändert. Für die Wahl des Präsidenten ist die absolute Mehrheit der abgegebenen gültigen Stimmen erforderlich (Vfs Art. 64 f.). Erreicht kein Kandidat diese Mehrheit, so entscheidet der Kongreß die Wahl zwischen den beiden stimmstärksten Kandidaten des ersten Wahlgangs. Der Kongreß wird nach Verhältniswahl lose gebundener Liste gewählt ${ }^{13}$. Das Wahlgebiet ist in Wahlkreise

9 S. dazu Enrique Evans, La delegación de facultades legislativas, in: Eduardo Frei u. a., Reforma constitucional 1970, Santiago 1970, S. 109-154.

10 S. dazu Tabelle 2, S. 75, die die Entwicklung der drei Blöcke nach Stimmstärken aufzeigt und deutlich macht, das von wenigen Ausnahmen abgesehen kein Block eine absolute Mehrheit der Stimmen erreichte. In den Fällen, in denen es seit 1932 zutraf, war entweder die Mitte vielfach gespalten (1953) oder bilIn den Fällen, in denen es seit 1932 zutraf, war entweder die Mitte vielfach gespalten (1953) oder bil-
dete keine Einheit aufgrund des Gegensatzes von Christdemokraten und Radikalen (1965), der beiden großen Parteien der Mitte.

11 Allein das Projekt einer Verfassungsreform konnte im Konfliktfall durch Volksentscheid entschieden werden; s. VfsArt. 109. In der Praxis hat dieser Artikel keine Anwendung gefunden, da ein Konflikt erst gegeben war, wenn eine Zweidrittelmehrheit des Kongresses gegen den Präsidenten votierte.

12 Die Wirksamkeit dieses Instruments wurde erst jüngst deutlich, als die Opposition gegen Allende während der Streikwelle im Oktober 1972 zu gleicher Zeit vier Ministeranklagen wegen verfassungswidrigen Verhaltens einbrachte. Allende mußte seine Regierung umbilden.

13 Zur Terminologie s. Bernhard Vogel, Dieter Nohlen, Rainer-Olaf Schultze, Wahlen in Deutschland. Theorie-Geschichte-Dokumente, 1848-1970, Berlin-New York 1971, S. 26-53. 
verschiedener Größe eingeteilt, in denen heute zwischen zwei und 18 Abgeordnete gewählt und die Mandate nach der Methode d'Hondt vergeben werden. Die Wahlkreiseinteilung fußt auf dem Zensus von 1920 und wurde seit 1932, sieht man von der Erhöhung der Mandatszahl von $1925=132$ auf heute $150 \mathrm{ab}$, nicht verändert. Die Nichtanpassung der Wahlkreise an die Bevölkerungsverschiebungen hat zu ähnlich krassen Mißverhältnissen in der Repräsentation geführt, wie sie das Deutsche Kaiserreich von 1871 kannte $^{14}$. Auch für das Abgeordnetenhaus herrscht heute praktisch das Prinzup territorialer Repräsentation vor, das für die Wahl des Senats gilt. Für die Bestellung dieser Kammer bilden mehrere Provinzen zusammen einen Wahlkreis, und unabhängig von der Bevölkerungszahl werden jeweils fünf Senatoren nach Verhältniswahl lose gebundener Liste und d'Hondt gewählt.

Die Hauptlinien der Verfassungsentwicklung seit 1925 bilden zum einen die Integration des Volkes in das politische System mittels Ausweitung des Wahlrechts und politischer Mobilisierung der Wählerschaft, zum anderen der Versuch, die Macht des Präsidenten im Verfassungssystem weiter zu stärken und bei einem sich ausweitenden staatlichen Funktionsbereich die Widerstände bei der Induktion sozialer und ökonomischer Transformation, die das Parlament der Exekutive leistete, aus dem Wege zu räumen. Beide Entwicklungstendenzen stehen in enger Verbindung mit der sozio-ökonomischen Entwicklung Chiles in den Jahrzehnten seit 1925.

\section{Sozio-ökonomischer Wandel seit 1925}

Seit die heute gültige Verfassung Chiles in Kraft getreten ist, hat sich in Chile ein grundlegender sozio-ökonomischer Wandel vollzogen, den wir im folgenden in seinen Hauptlinien nachzeichnen und auf seine Folgen hin untersuchen wollen.

Noch 1935 konnte Chile als Agrarland charakterisiert werden, in welchem die Mehrheit der Bevölkerung auf dem Lande lebte, die Mehrheit der Beschäftigten auf dem Lande arbeitete und die Politik Chiles von den Großgrundbesitzern bestimmt wurde ${ }^{15}$. Diese Aussagen trafen spätestens seit den sechziger Jahren nicht mehr zu. Bereits im Jahre 1940 machte die städtische Bevölkerung 52,5 Prozent aus. Sie stieg seither kontinuierlich und umfaßte im Jahre 1970 74,2 Prozent ${ }^{16}$. Die Landwirtschaft verlor in den frühen fünfziger Jahren ihre Position als erster Beschäftigungssektor an den tertiären Bereich Handel und Dienstleistungen; ihr Ver-

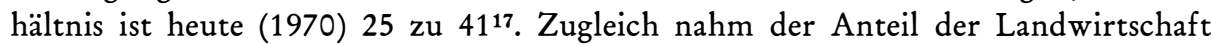
am Volkseinkommen Chiles kontinuierlich ab. Dies spiegelt den ökonomischen Bedeutungsverlust jenes Wirtschaftszweiges, mit dem sich traditionellerweise die politische Macht in Chile verband, deutlich wider. Die Landwirtschaft trug 1970 nur mehr mit 7,2 Prozent zum Volkseinkommen bei ${ }^{18}$. Allein die politische Reprä-

\footnotetext{
14 Die extremen Differenzen werden bereits bei einem Vergleich nur der Wahlkreise von Groß-Santiago deutlich: Wahlkreis 1 mit etwa 600000 Einwohnern bestellt 18 Abgeordnete. Wahlkreis 2 mit etwa 900000 Einwohnern und Wahlkreis 3 mit über 1,4 Mill. Einwohnern wählen jeweils fünf Abgeordnete (Zahlen für 1969); s. dazu demnächst meinen Beitrag Chile (s. Anm. 3). Zur Situation im angegebenen Beispiel des Deutschen Kaiserreichs s. Bernhard Vogel, Dieter Nohlen, Rainer-Olaf Schultze, Wahlen in Deutschland (s. Anm. 13), S. 98-104.

15 Jorge M. McBride, Chile, su tierra, 2. Aufl. Santiago 1970, S. 33

15 La Economía de Chile en el período 1950-1963, hrsg. vom Instituto de Economía der Universidad de Chile, 2 Bde., Santiago 1963, 2. Bd., S. 11; CELADE-ODEPLAN, Proyecciones de la Población de Chile, 1960-2000, Santiago 1968.

17 In Prozent der beschäftigten Bevölkerung; s. ODEPLAN, Plan de la Economía Nacional. Antecedentes sobre el desarrollo chileno 1960-70, Santiago 1971, S. 66.

18 Comentarios sobre la situación económica, hrsg. von der Universidad de Chile, Facultad de Ciencias Económicas, Santiago 1972, S. 39.
} 
sentation des dünn besiedelten Landes blieb aufgrund der unveränderten Wahlkreiseinteilung zum Nachteil der Ballungszentren aufrechterhalten.

Doch konnte auf diese Weise die Vormachtstellung der Landbesitzer in der chilenischen Politik nicht konserviert werden. Entscheidendes Merkmal dafür und zugleich für die fundamentalen Veränderungen im politischen Kräftefeld bildet die unter der Regierung Frei gegen den erbitterten Widerstand der politischen Rechten durchgesetzte Agrarreform. Sie leitete eine radikale Umverteilung des Bodens ein, welche die Regierung Allende bis Jahresende 1972 in dem Umfang, den das Gesetz vorsah, und darüber hinaus zum Abschluß gebracht hat ${ }^{19}$.

Die wesentlichen dynamischen Faktoren der sozio-ökonomischen Veränderung waren eine Beschleunigung der Bevölkerungszunahme (seit Jahrhundertbeginn ist die Bevölkerung Chiles auf das Dreifache angewachsen) ${ }^{20}$, eine Intensivierung der Verstädterung ${ }^{21}$ und eine staatlich induzierte Industrialisierung des Landes. Die jährliche industrielle Zuwachsrate in den Jahren 1914 bis 1964 lag mit einem Mittelwert von 4,3 Prozent weit über dem Wachstum der Gesamtwirtschaft ${ }^{22}$. Doch verlief die Industrialisierung, die importsubstitutiv angelegt war und der deshalb die eigene Dynamik und nationale Projezierung fehlten ${ }^{23}$, in vielerlei Hinsicht ungenügend. Chile blieb vom Import von Industrieprodukten und technischem Know-how abhängig. Diese Situation wurde durch die Struktur seiner Exportwirtschaft noch wesentlich verschärft. Über 85 Prozent der chilenischen Ausfuhren machen Rohstoffe des sog. extraktiven Bereichs aus, die der Verschlechterung der „terms of trade" und der Preisinstabilität unterliegen. Somit ist die interne Investitionsrate von externen Bedingungen abhängig. Da Chile aufgrund der Stagnation der Landwirtschaft von einem Export- zu einem Importland agrarwirtschaftlicher Produkte wurde, verschärfte sich noch seine Außenhandelssituation.

Mitverantwortlich für die Mängel im Industrialisierungsprozeß waren Unlust und Unvermögen der chilenischen Mittelschicht, unternehmerische Initiativen zu entwickeln. Sie suchte die Anstellung in der Verwaltung, die risikolos einen relativ leichten sozialen Aufstieg versprach ${ }^{24}$. Die staatliche Industrialisierungspolitik der ersten Volksfrontregierungen Chiles unter Führung der Radikalen besaß deshalb in der Schaffung von staatlichen Positionen für eine sich ausweitende Mittelschicht Santiagos ein wesentliches Motiv. Einerseits entstand eine aufgeblähte, wenig effiziente staatliche Verwaltung ${ }^{25}$, andererseits kein unabhängiges Bürgertum unternehmerischer Qualitäten, das an Leistung und Produktivität orientiert war ${ }^{26}$ und sich von der Bindung an die Oberschicht des Landes ökonomisch, sozial und sozialpsychologisch freimachte. Neben der psychischen Mobilisierung ${ }^{27}$ blieb auch eine politische, strukturreformerische Mobilisierung aus. Zwar konnte die Mittel-

19 S. dazu ODEPLAN, Informe económico anual, 1971, Santiago 1971, S. 94 f. Eine Analyse der Agrarpolitik der Regierungen findet sich bei Dieter Nohlen, Chile - das sozialistische Experiment, Hamburg 1973, Kapital II, 2.4 und IV, 1.5

20 1912: 3,4 Mill., 1930: 4,3, 1960: 7,7, 1970: 9,8 Mill. Einwohner; nach Armand Mattelart, Manual de Análisis Demográfico, Santiago 1964, S. 121 und ODEPLAN, Plan de la Economía Nacional (s. Anm. 17), S. 294.

21 ODEPLAN, Plan de la Economía Nacional (s. Anm. 17), S. 295.

22 Oscar Muñoz, Crecimiento Industrial de Chile, 1914-1965, 2. Aufl., Santiago 1971, S. 25 f.

23 Aníbal Pinto, Política y desarrollo, Santiago 1968, S. 32-34.

24 Germán Urzúa Valenzuela/Anamaria García Barzelatto, Diagnóstico de la burocracia chilena (1818-1969), Santiago 1971, S. 162.

25 Siehe dazu die vorangenannte Untersuchung der chilenischen Verwaltung von Germán Urzúa Valenzuela und Anamaria García Barzelatto.

26 Víctor Brodersohn, Sobre el carácter dependiente de la burguesía industrial, in: Chile hoy, Mexiko 1970, S. 322-344, arbeitet diesen Aspekt besonders heraus. Interessant in diesem Zusammenhang ist die Meinung des Sozialisten Clodomiro Almeyda über die zu wenig kapitalistische Orientierung und Prägung der chilenischen Mittelschicht, in Reflexiones políticas, Santiago 1958, S. 72.

$27 \mathrm{Zu}$ diesem Begriff und $\mathrm{zu}$ dem mit ihm verbundenen Entwicklungskonzept s. die inzwischen klassische Studie von Everett E. Hagen, On the Theory of Social Change. How Economic Growth Begins, Dorsey Press 1962. 
schicht bei der Suche nach individuellen Privilegien ihren Lebensstandard anheben und moderne Konsumansprüche entwickeln und teilweise befriedigen, sie wich aber der Auseinandersetzung mit den alten Führungseliten aus. Bezeichnend dafür war, daß Reformen wie eine Agrarreform, die die Radikalen und die Rechte entzweit hätte, von vornherein unterblieben. Eine substantielle Veränderung der Gesellschaftsstruktur stand deshalb außerhalb ihrer politischen Zielsetzunger. In ihrer Harmonisierungstenden $z^{29}$ assimilierte die Mittelschicht vielmehr die Bergbau- und Industriearbeiterschaft, die Gewerkschaften und die marxistischen Parteien an das politische System, während die Landarbeiter unverändert direkt von der landbesitzenden Oberschicht kontrolliert wurden. Hier ist wichtig festzuhalten, daß auch die politische Ideologie der Linken bestimmte Arbeitergruppen favorisierte und für sie spezifische Privilegien zu erreichen suchte ${ }^{30}$. Die Volksfrontpolitik vertiefte die sozialen Differenzen zwischen Mittelschicht und (Lumpen-)Proletariat, indem die Modernisierung ausgerichtet wurde auf die Befriedigung der (Konsum-)Interessen der Mittelschicht und auf Kosten der unteren Schichten ablief. Die Mittelschicht begründete alsdann ein neues politisches Zusammengehen mit den Vertretern der chilenischen Oberschicht, das nach dem populistischen Zwischenspiel des Generals Carlos Ibañez del Campo (1952-1958) in der Präsidentschaft des rechtsunabhängigen Jorge Alessandri Rodriguez (1958-1964) ihren Höhepunkt fand.

Eine weitere Folge der Industrialisierungspolitik der Volksfront war, daß von seiten des Staates nicht nur eigene Betriebe für Eisen, Erdöl, Elektrizität etc. aufgebaut und weiter gefördert wurden, sondern auch private Unternehmen gestützt wurden. Der Staat übernahm die Funktion, der stark monopolisierten, obligarchisch strukturierten Privatindustrie ${ }^{31}$ entscheidende finanzielle Assistenz zu leisten $^{32}$. Im Jahre 1970 erzeugte der Staat in seinen Unternehmungen etwa 40 Prozent des Bruttoinlandprodukts, finanzierte aber 70 Prozent der im Lande vorgenommenen Investitionen, ohne die Kontrolle der aus öffentlichen Geldern resultierenden Wirtschaftstätigkeit in Anspruch zu nehmen ${ }^{33}$. Diese Politik führte zu einer engen Verbindung des Staates mit der Großbourgeoisie, die ihrerseits den Staat zum Schutz bestimmter privater Interessen einsetzte und als Instrument zur Aufrechterhaltung des gesellschaftlichen Status quo begriff. Die Interessen der Oberschicht und der Mittelschicht waren somit eng mit dem Staat verknüpft ${ }^{34}$. Zwischen beiden Schichten bestand eine gewisse Mobilität. Diese Tatsache verschaffte den Eindruck einer offenen Gesellschaft. Sie traf jedoch nur für die gesamtgesellschaftlich privilegierten Schichten $\mathrm{zu}^{35}$. Darauf gründete sich die hervorragende Rolle, die die traditionellen Parteien, Konservative, Liberale und Radi-

28 Vgl. die lateinamerikanische Perspektive dieses Phänomens bei Rodolfo Stavenhagen, Siete tésis equivocadas sobre América Latina, in: F. H. Cardoso/F. Weffort: América Latina. Ensayos de interpretación sociológico-política, Santiago 1970, S. 82-94, hier S. 89-92.

29 S. dazu John J. Johnson, The political role of the Latin American Middle Sectors, in: Annuals 334/1961, S. 25.

30 Osvaldo Sunkel, Cambio y frustración en Chile, in: Claudio Veliz, Obstáculos para la transformación de América Latina, Mexiko 1969, S. 112-144, hier S. 130.

31 Zur Monopolisierung der chilenischen Volkswirtschaft s. vor allem Ricardo Lagos, La industria en Chile. Antecedentes estructurales, Santiago 1966, zur oligarchischen Tendenz in den Unternehmen die Untersuchung von Genaro Arriagada, La oligarquía patronal chilena, Santiago 1970. Einen guten Ưberblick gibt die Studie von Sergio Aranda und Alberto Martínez, Estructura económica: Algunas características fundamentales, in: Chile hoy (s. Anm. 26), S. 55-172.

32 Vgl. Víctor Brodersohn, Sobre el carácter dependiente (s. Anm. 26), S. 337

33 Zur Entwicklung des öffentlichen Anteils am Investitionsvolumen s. David F.elix, Desequilibrios estructurales y crecimiento industrial, Santiago 1958, Tabelle 2, und CEDEM, Elementos para un análisis de la intervención del Estado en la economía chilena, Santiago 1968, Tabellen 1-3.

34 James Petras, Politics and Social Forces in Chilean Development, University of California Press, Berkeley and Los Angeles 1969, S. 342 f.

35 Osvaldo Sunkel, Cambio y frustración (s. Anm. 30), S. 129. 
kale, in enger Interessenverbindung mit dem in Chile höchst profitabel arbeitenden ausländischen Kapital ${ }^{36}$ bis in die sechziger Jahre hinein zu spielen vermochten, begünstigt auch durch die Vorherrschaft der Interessen von Banken, Industrie und Handel in den Kommunikationsmedien ${ }^{37}$ und nicht zuletzt durch die bis dahin konservative Haltung der katholischen Kirche.

Der sozio-ökonomische Wandel, der auf dem raschen Bevölkerungswachstum und der beschleunigten Urbanisierung fußte ${ }^{38}$, war, da die Industrialisierung auf halbem Wege mit zunehmend dysfunktionalen Folgen für den gesellschaftlichen Strukturwandel verharrte, in Umfang und Qualität nicht ausreichend, um Chile ökonomisch zu entwickeln und gesellschaftlichen Pluralismus und soziale Mobilität entstehen zu lassen. So verstärkten sich nur die bestehenden Strukturen; der traditionelle, in einem einzigen historischen Prozeß entstandene sozio-ökonomische Dualismus des Landes ${ }^{39}$ nahm zu. Er berechtigt dazu, von zwei nebeneinander bestehenden Gesellschaften zu sprechen, deren entscheidende Gemeinsamkeit ihre Genesis ist. Die moderne Gesellschaft lebt in den industriellen Zentren, in denen sich die Bevölkerung konzentriert und zu denen die Binnenwanderung weiter anhält. Hier herrschen beachtliche Einkommensanstiege vor, Märkte für Industrieprodukte, starke Gewerkschaften, die Lohnforderungen etc. durchzusetzen in der Lage sind, und ein gehobener Bedarf an Konsum- und Gebrauchsgütern. Die andere, traditionelle Gesellschaft lebt in den dünn besiedelten ländlichen Gebieten, aber auch aus ihr herausgerissen auf der gleichen Entwicklungsstufe als Marginalbevölkerung in Elendssiedlungen um die großen Städte herum. Hier herrschen Niedrigeinkommen, Wirtschaftsformen subsistenzwirtschaftlichen Charakters, Märkte vormoderner Produkte, schwacher gesellschaftlicher Organisationsgrad und offene wie verdeckte Arbeitslosigkeit vor.

Die interne Polarisierung der chilenischen Gesellschaft ${ }^{40}$ und der schwache Integrationsgrad der chilenischen Volkswirtschaft, wichtige Kriterien für den Entwicklungsstand eines Landes ${ }^{41}$, und ihre Fremdbestimmung durch externe Handlungsträger machten, wenn man die vorzeitig stagnierte ökonomische Entwicklung und gesellschaftliche Mobilität überwinden wollte, spätestens mit Ende der Präsidentschaft Alessandris im Jahre 1964 dringende Strukturreformen erforderlich. Dies war der Boden für die grundlegenden Veränderungen im Parteiensystem Chiles, die sinnfällig in der Alternative bei den Präsidentschaftswahlen von 1964 zum Ausdruck kamen. Es kandidierten der Christdemokrat Eduardo Frei Montalva mit einem Programm der „Revolution in Freiheit" und Salvador Allende Gossens mit einem sozialistischen Programm der marxistischen Parteienkoalition FRAP aus

36 S. dazu Orlando Caputo und Roberto Pizarro, Imperialismo, dependencia y relaciones económicas inter. nacionales, Santiago 1970, auch den Aufsatz beider Autoren, in: Chile hoy (s. Anm. 26): Dependencia e inversión extranjera, S. 173-209. Allgemein zur Problematik der ausländischen Kapitalinvestitionen in Lateinamerika: Miguel S. Wionczek, Lateinamerika und das ausländische Kapital, Hamburg 1969.

37 Dazu liegen verschiedene Untersuchungen vor, die im Sonderheft der Cuadernos de la Realidad Nacional, Nr. 3, veröffentlicht wurden: Armand Mattelart, Mabel Piccini, Michèle Mattelart, Los medios de comunicación de masas. La ideología de la prensa liberal en Chile, Santiago 1970.

38 Die Urbanisierung gilt in den Studien zur sozialgeschichtlichen Entwicklung Lateinamerikas als wichtiger wenn nicht sogar vorrangiger Faktor. S. etwa Kenneth F. Johnson, Causal factors in Latin American political instability, in: The Western Political Quarterly, XVII/1964, S. 432-446. Vielfach wurde die Variable „Urbanisierung “ zur Messung des sozialen Wandels herangezogen. Für Chile haben John Friedmann und Thomas Lackington die These aufgestellt, daß die übermäßige Urbanisierung die ökonomische Entwicklung des Landes behindert habe. S. J. Friedmann/Th. Lackington, La hiperurbanización y el Entwicklung des Landes behindert habe. S. J. Friedmann/Th. Lackington, La hiperurbanización y el 39 S. Rodolfo Stavenhagen, Siete tesis (s. Anm. 28), S. 84.

$40 \mathrm{Zu}$ Begriff und Problem der internen Polarisierung s. jetzt in leicht zugänglicher Form Osvaldo Sunkel, Transnationale kapitalistische Integration und nationale Desintegration: Der Fall Lateinamerika, in: Dieter Senghaas, Imperialismus und strukturelle Gewalt. Analysen über abhängige Reproduktion, Frankfurt am Main 1972, S. 258-315, hier bes. S. 268-278.

$41 \mathrm{Vgl}$. Reimut Jochimsen, Dualismus als Problem der wirtschaftlichen Entwicklung, in: Bruno Fritsch (Hrsg.), Entwicklungsländer, Köln-Berlin 1968, S. 65-82. 
Kommunisten und Sozialisten, also nur Bewerber der sog. partidos modernizantes ${ }^{42}$. Der Kandidat der Radikalen Partei besaß keine Siegchance, zumal nachdem sich Konservative und Liberale aus dieser Erkenntnis heraus von ihm abgewandt hatten und die demokratische Reformalternative zur Abwehr der marxistischen Revolution $^{43} \mathrm{zu}$ unterstützen bereit waren.

\section{Entwicklung von politischer Partizipation und Parteiensystem}

Der Aufstieg der neuen Parteien, die sich von den traditionellen Parteien nicht nur in programmatischer, sondern vor allem in organisatorischer Hinsicht unterscheiden, verläuft in etwa synchron mit der „Fundamentaldemokratisierung“ (Karl Mannheim) des politischen Systems, die in Chile erst in den sechziger Jahren stattfand. Die Integration der unteren Schichten und jener Bevölkerungsteile, denen die Verfassung von 1925 die Beteiligung am politischen Prozeß als Aktivbürger verwehrte, wurde durch die Ausweitung des Wahlrechts auf alle über 18 Jahre alten Chilenen beiderlei Geschlechts herbeigeführt. Die Frauen erhielten durch das Gesetz Nr. 9292 vom 14. Januar 1949 das Wahlrecht eingeräumt, nachdem sie bereits seit $1935 \mathrm{zu}$ den Kommunalvertretungen hatten wählen können. Durch die Verfassungsreform vom 23. Januar 1970 (Gesetz Nr. 17.284) wurden das Wahlalter von 21 Jahren auf 18 Jahre gesenkt und das Wahlrecht auch den Analphabeten zuteil44. Eine Bestimmung indes blieb aufrechterhalten, die für die effektive Ausweitung des Wahlrechts große Bedeutung besaß. Zur Stimmberechtigung ist die Eintragung in die Wählerregister notwendig. Dieses Erfordernis, das durch das Wahlgesetz vom 16. Mai 1962 (Nr. 14.852) für alle Chilenen im wahlfähigen Alter zur Pflicht erhoben wurde, hat den tatsächlichen Rhythmus bestimmt, in dem sich die Partizipation der Bevölkerung am politischen Prozeß ausdehnte. Im Jahre 1952 waren trotz allgemeinem Wahlrecht nur effektiv 38,0 Prozent der potentiellen Wählerschaft wahlberechtigt. Dieser Prozentsatz konnte sich bis 1965 auf 83,0 Prozent erhöhen. Erst in den sechziger Jahren konstituierte sich in Chile ein Wahlkörper, der einer Wählerschaft nach allgemeinem Wahlrecht vergleichbar ist.

Die Entwicklung der Parteien und des Parteiensystems konnte infolge der Ausweitung der Partizipation von den wirtschaftlichen und gesellschaftlichen Veränderungen und Problemen zunehmend stärker beeinflußt werden. Dabei ist allerdings nicht von einer einfachen positiven Korrelation zwischen Ausweitung der Wählerschaft und Stimmenzuwachs reformerischer, die Interessen der unteren Schichten der Bevölkerung artikulierender Parteien auszugehen. Guillermo Briones hat in seiner Untersuchung über soziale Schichtung und Parteipräferenz im Wahlgebiet

42 Darunter werden die Christdemokraten, die Sozialisten und die Kommunisten verstanden, deren Geschichte mit Ausnahme der Kommunistischen Partei nicht vor Inkrafttreten der Verfassung von 1925 zurückreicht. Zu ihrer Gesamtstimmentwicklung im Vergleich zu den traditionellen Parteien (Konservative, Liberale und Radikale) s. Tabelle 2, S. 75.

43 Den vieldeutigen Begriff der "Revolution “ verwende ich zur Kennzeichnung grundlegender Veränderungen der politischen oder sozio-ökonomischen Struktur eines Landes. Die Frage der Gewaltsamkeit un' der Rapidität des Prozesses stelle ich hintan. Der Revolutionsbegriff wird am Ausmaß und an dt. Intensität des von den politischen Gruppen intendierten oder durchgeführten Wandels orientiert, die Methode, ob gewaltsam oder friedlich-geregelt, zu einer Funktion der sozio-ökonomischen und politischer Transformation. Je intensiver und umwälzender Absichten und Maßnahmen der Veränderung, desto bf rechtigter ist die Verwendung des Begriffes "Revolution“.

44 Eine der wenigen Studien zum Problemkreis Analphabetismus und Wahlrecht stellt die Arbeit von Gustavo Sarriego-McGinty, Los derechos políticos de los analfabetos, Santiago 1966, dar, die besonders auf die Verhältnisse in Ćhile eingeht. Aufgrund des Rückgangs des Analphabetismus in Chile von 20,0 Prozent im Jahre 1950 auf unter 10,0 Prozent im Jahre 1970 nahm die praktische Relevanz dieser restriktiven Bestimmung für den chilenischen Wahlkörper kontinuierlich ab. 
von Groß-Santiago für das Jahr 1958 nachgewiesen ${ }^{45}$, daß die Personen im wahlfähigen Alter, die nicht wahlberechtigt waren und in größerem Maße unteren Schichten angehörten, keineswegs eine verstärkte Linksorientierung besaßen. Deshalb erlangte die verzögerte Partizipation großer Bevölkerungsteile nie parteipolitische Brisanz. Wie auch die jüngsten Präsidentschaftswahlen zeigen, bei denen der Rechtskandidat Alessandri 35,3 Prozent der Stimmen auf sich vereinigen konnte ${ }^{46}$, ist das Wahlverhalten der Chilenen weniger schichtenspezifisch determiniert. Ebensowenig spielen unterschiedliche ökonomische Entwicklungen der Regionen eine dominierende Rolle, wie Atilio Borón aufzeigen konnte ${ }^{47}$. Vielmehr hängt die Wählerpräferenz für die neuen, und hier vor allem für die marxistischen Parteien, in hohem Maße vom Organisationsgrad der Wählerschaft ab. Kommunisten und Sozialisten erzielten dort erhebliche Stimmenanteile, wo die Gewerkschaftsbewegung erfolgreich Fuß gefaßt hatte ${ }^{48}$. Auf dem Lande und in den randstädtischen Siedlungen dagegen blieben sie bis in die Gegenwart schwach, auch als dort hohe Partizipationsziffern erreicht wurden. Einerseits bestanden die traditionalen Strukturen in den dünnbesiedelten Gebieten und die mit ihnen zusammenhängenden sozio-psychologischen Verhaltensweisen der Bevölkerung bis in die Mitte der sechziger Jahre fort und begünstigten traditionelles Wählerverhalten; andererseits bemühten sich die marxistischen Parteien kaum um die Lösung sozialer Probleme dieses Bevölkerungsteiles und um den Aufbau von Organisationen der Bauern und den Gewinn einer sozialen Basis in jenen Zonen. Erst die christdemokratische Agrarreform und die erfolgreiche Politik dieser Partei, die unterprivilegierte Bevölkerung zu organisieren ${ }^{49}$, haben in Verbindung mit den erhöhten Partizipationschancen in der Gegenwart neue Voraussetzungen für die modernen Parteien auf dem Lande geschaffen.

Die sozial- und wirtschaftsstatistischen Daten der Tabelle 1 zeigen, wie eng der Aufstieg der neuen Parteien mit den sozio-ökonomischen Veränderungen seit 1925 verbunden sind. Die Auswahl der Daten verdeutlicht, daß die entscheidenden Schritte zu einer grundlegenden Veränderung des Parteiensystems zwischen 1937 und 1941 durch den Wahlsieg der Volksfront und zwischen 1961 und 1965 durch den Wahlsieg des Christdemokraten Eduardo Frei erfolgten. Besonders die rapide Stimmenzunahme dieser jüngsten sozialrevolutionären Partei unter den partidos modernizantes ${ }^{50}$ war eine Folge der Industrialisierungspolitik unter Führung der Radikalen Partei, denn ihre Wählerschaft rekrutierte sich aus dem neuen Mittelstand: Angestellten, Kleinhandel, akademischen Berufen und Facharbeitern in den urbanen Gebieten ${ }^{61}$. Die Christdemokraten konnten ihren Stimmenanteil bei den Wahlen von 1961, bei denen sie 16,0 Prozent erhielten, auf 43,6 Prozent im Jahre 1965 erhöhen. Die neuen Parteien konnten insgesamt zwei Drittel der Stimmen auf

45 Guillermo Briones, La estructura social y la participación política, in: Hernán Godoy, Estructura (s. Anm. 38), S. 476-488, hier S. 479-482.

46 Zur Interpretation der Wahlen von 1970 s. Dieter Nohlen, Chile (s. Anm. 19), Kapitel III, 3.

47 Atilio Borón, Desarrollo económico y comportamiento político, in: Revista Latinoamericana de Ciencia Política, I/1970, S. 236-287.

48 Vgl. dazu bereits Ricardo Ċruz-Coke, Geografía electoral de Chile, Santiago 1952, S. 75-85. Vgl. auch Jorge Barria S., Los sindicatos de la Gran Minería del Cobre, Santiago 1970, S. 154-160.

49 Die Christdemokraten maßen der Organisierung der Landarbeiter erhebliche Bedeutung im Prozeß der

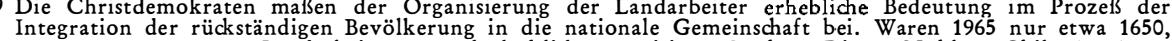
so 1970 etwa 145000 Landarbeiter gewerkschaftlich organisiert. S. dazu Dieter Nohlen, Chile (s. Anm. 19), Kapitel II, 1 und 2.5 .

$50 \mathrm{Zur}$ Geschichte und Ideologie dieser Parteien s. Hernán Ramírez Necochea, Orígen y formación del partido Comunista de Chile, Santiago 1965; Julio Cesar Jobet, El Partido Socialista de Chile, 2. Bde., 2. Aufl., Santiago 1971; Jaime Castillo Velasco, Las fuentas de la Democracia Cristiana, 2. Aufl., Santiago 1968; George W. Grayson, The Chilean Christian Democratic Party: Genesis and Development, 1967, spanischsprachige Ausgabe El Partido Demócrata Cristiano Chileno, Buenos Aires 1968.

51 Vgl. Enzo Faletto, Eduardo Ruiz, Conflicto político y estructura social, in: Chile hoy (s. Anm. 26), S. 213-254, hier bes. S. 214-224. 
sich vereinigen. Die hohe Flexibilität im chilenischen Wählerverhalten, die im Durchbruch der neuen Parteien zum Ausdruck kommt, wurde dabei durch die relativ späte Konstituierung eines Wahlkörpers nach allgemeinem Wahlrecht begünstigt.

Tabelle 1: Sozio-ökonomische und politische Indizes des Wandels 1925, 1937/1941, $1961 / 1965$

\begin{tabular}{|c|c|c|c|c|c|c|c|c|}
\hline \multirow[t]{2}{*}{$\mathrm{Ja}$} & \multirow{2}{*}{$\begin{array}{l}\text { Urbanisierung } \\
\text { 0\% der städt. } \\
\text { Bevölkerung }\end{array}$} & \multicolumn{2}{|c|}{$\begin{array}{c}\text { Beschäftigte } \\
\text { in } \%\end{array}$} & \multirow{2}{*}{$\begin{array}{c}\text { Wahl- } \\
\text { berech- } \\
\text { tigte } \\
\text { in } \% \text { der } \\
\text { Bevöl- } \\
\text { kerung }\end{array}$} & in $\%$ & in $\%$ & \multicolumn{2}{|c|}{$\begin{array}{l}\text { Stimmen- } \\
\text { anteile }\end{array}$} \\
\hline & & $\begin{array}{l}\text { Land- } \\
\text { wirt- } \\
\text { schaft }\end{array}$ & $\begin{array}{l}\text { Tert. } \\
\text { Sektor }\end{array}$ & & $\begin{array}{c}\text { der } \\
\text { Wahl- } \\
\text { berech- } \\
\text { tigten }\end{array}$ & $\begin{array}{c}\text { der } \\
\text { Bevöl- } \\
\text { kerung }\end{array}$ & 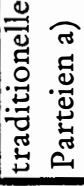 & 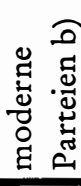 \\
\hline 1925 & $46,4-49,4 c)$ & & & 7,7 & 86,6 & 6,4 & 73,6 & d) \\
\hline 1937 & $49,4-52,5$ e) & & & 10,0 & 86,7 & 8,7 & 70,6 & 15,4 \\
\hline $1941 \mathrm{f})$ & 52,5 & 37,0 & 32,0 & 11,4 & 78,2 & 8,9 & 51,9 & 37,3 \\
\hline $1961 \mathrm{f})$ & 68,2 & 31,0 & 36,0 & 23,8 & 74,5 & 17,8 & 56,9 & 34,9 \\
\hline 1965 & 71,5 & 27,0 & 37,0 & 34,1 & 80,3 & 27,4 & 26,5 & 66,9 \\
\hline
\end{tabular}

Anmerkungen: a) Traditionelle Parteien: Konservative, Liberale und Radikale; b) Moderne Parteien (partidos modernizantes $=$ Reformparteien) Kommunisten, Sozialisten und Christdemokraten; c) die beiden Daten entsprechen den Jahren 1920 und 1930; d) Vorläufer der Kommunisten und Sozialisten war die Demokratische Partei, die 1925 einen Stimmenanteil von 22,3 Prozent besaß; e) die beiden Daten entsprechen den Jahren 1930 und 1940; f) die Daten der ersten drei Rubriken gelten jeweils für die Jahre 1940 und 1960.

(Quellen: Armand Mattelart, Manual de Análisis Demográfico, Santiago 1964; Jorge Tapia V., Bureaucratic Power in a Developing Country, Diss. University of Texas, Austin 1969; ODEPLAN, Plan de la Economía Nacional. Antecedentes sobre el desarrollo chileno 1960-70, Santiago 1971; Dirección del Registro Electoral, Santiago).

Christliche Demokraten, Kommunisten und Sozialisten propagierten in Anbetracht der gesellschaftlichen Folgen der Industrialisierungspolitik und der ökonomischen Stagnation des Landes eine neue Politik struktureller Veränderungen in Wirtschaft und Gesellschaft. Zur Überwindung von Unterentwicklung und außenwirtschaftlicher Abhängigkeit forderten sie eine Agrarreform, die Nationalisierung strategischer Industrien, Einkommensumverteilung, Bildungsreform etc. Mit ihren revolutionären Programmen wurde die Frage der Reform der Verfasung als Voraussetzung erfolgreicher sozio-ökonomischer Transformation zu einem zentralen Gegenstand der innenpolitischen Diskussion. 
Tabelle 2: Die Entwicklung des chilenischen Parteiensystems nach dem LinksRechts-Kontinuum 1925-1969 a)

\begin{tabular}{l|c|ccc|r} 
Jahr & $\begin{array}{c}\text { Partizipation } \\
\text { in \% der } \\
\text { Bevölkerung }\end{array}$ & \multicolumn{3}{|c}{ Stimmenanteile der Parteien } \\
\hline 1925 & Rechts & Mitte & Links & Andere b) \\
1932 & 6,6 & 52,3 & 21,3 & 22,4 & 3,9 \\
1937 & 7,3 & 36,9 & 25,9 & 21,4 & 15,8 \\
1941 & 8,7 & 47,9 & 18,7 & 24,7 & 8,7 \\
1945 & 8,9 & 32,9 & 26,4 & 37,0 & 3,6 \\
1949 & 8,4 & 45,7 & 22,6 & 28,4 & 3,3 \\
1953 & 8,2 & 42,1 & 40,8 & 16,1 & 1,1 \\
1957 & 12,9 & 22,0 & 54,6 & 19,7 & 3,7 \\
1961 & 12,3 & 29,1 & 49,6 & 18,1 & 2,0 \\
1965 & 17,8 & 31,4 & 38,1 & 30,1 & 0,5 \\
1969 & 27,4 & 13,6 & 58,3 & 27,8 & 0,3 \\
& 24,9 & 20,4 & 47,6 & 31,7 & 0,1 \\
\hline
\end{tabular}

Anmerkungen: a) Stimmanteile der Parteien bei den Parlamentswahlen. Es würde zu weit führen, die Maßstäbe für die Einteilung der Parteien in die Gruppen Rechts, Mitte und Links hier offenzulegen. Die Eingruppierung erfolgte in Anlehnung an O. Sunkel, P. Paz und O. Rodriguez, Antecedentes cuantitativos referentes al desarrollo de América Latina, 2 Bde., hekt., Santiago 1966, Bd. 2, S. 80-82. Die zentralen Parteien der Rechten sind Konservative und Liberale, seit 1967 bilden sie zusammen die Nationale Partei; der Mitte: Radikale und Christdemokraten; der Linken: Sozialisten und Kommunisten. Eine andere Untergliederung der Parteien in Rechte, Mitte und Linke auf der Basis der Parteienbündnisse für die Wahl des Präsidenten 1961-1969 findet sich bei Dieter Nohlen, Chile vor den Präsidentschaftswahlen. Westliche Demokratie und sozialer Wandel in Lateinamerika, in: Aus Politik und Zeitgeschichte, 35-36/1970, S. 32. Die kratie und sozialer Wandel in Lateinamerika, in: Aus Politik und Zeitgeschichte, 35-36/1970, S. 32. Die
Radikale Partei zählt dort 1961 und 1965 zur Rechten, 1969 zur Linken. Eine solche Zuordnung widerspricht zwar soziologischen Gesichtspunkten, hat aber gegenüber der oben unternommenen den Vorteil, die politischideologischen und programmatischen Standortwechsel der Parteien zu berücksichtigen. b) Vor allem Unabhängige.

(Quelle: Dirección del Registro Electoral).

\section{Die Ausweitung der Macht der Exekutive}

Die Reform der Verfassung war kein grundlegend neues innenpolitisches Thema. Fast alle Präsidenten haben auf die Notwendigkeit verwiesen, die Verfassung in den Punkten zu ändern, die die Macht der Exekutive in der Durchführung des mit einem direkten Wählermandat versehenen Programms begrenzten. Dieses Postulat erwuchs besonders aus der Tatsache, daß einerseits das chilenische Parteiensystem nur schwerlich eine Mehrheitsbildung im Kongreß zuläßt, die wiederum Bedingung einer erfolgreichen Gesetzgebungsarbeit ist, und daß sich andererseits die reformerischen Ideen und Bewegungen eher in der Wahl des Präsidenten durchsetzen als in der auf Proporz beruhenden Wahl des Kongresses. Von hier her lag nahe, entweder eine Einschränkung der Kompetenzen des Kongresses anzustreben oder eine Reform durchzuführen, die eine stärkere politische Übereinstimmung von Präsident und Parlament herbeiführte. Gedacht wurde daran, beide Organe zu gleicher Zeit wählen zu lassen, zumal die Bestellungsweise des Senats es praktisch unmöglich macht, daß ein Präsident, der nicht aus der mandatsstärksten Gruppierung des Kongresses hervorgeht, bei den nachfolgenden Wahlen zum Parlament eine Mehrheit in beiden Häusern des Kongresses erhält ${ }^{52}$. Derart grund- 
legende Veränderungen des politischen Systems, die den Einfluß des Präsidenten auf das Parlament hätten dominant werden lassen, hatten auch zu einer Zeit keine Aussicht, vom Kongreß angenommen zu werden, als solche Reformen die demokratisch-pluralistische Struktur des Regierungssystems nicht angetastet hätten.

Seit den sechziger Jahren besitzt die verfassungspolitische Frage eine stärkere gesellschafts- und entwicklungspolitische Motivation und Relevanz. Zentraler Gesichtspunkt ist dabei die Überzeugung der sozial fortschrittlichen Gruppen, daß das traditionelle Institutionensystem und die Kompetenzverteilung zwischen Exekutive und Legislative entweder den gesellschaftlichen Wandel hemme (so die Ansicht der Christdemokraten) oder überhaupt untauglich sei, eine wirkliche (und dann sozialistische) Entwicklung des Landes in wirtschaftlicher, gesellschaftlicher und kultureller Hinsicht zuzulassen (so die Meinung der Marxisten). Darauf ist gleich noch zurückzukommen.

Entwicklungs- und gesellschaftspolitisch kam der Verfassungsfrage vor allem deshalb erhöhte Bedeutung zu, weil der Staat in den vierziger und fünfziger Jahren kontinuierlich mehr Funktionen in der Wirtschaft und schließlich die Steuerung des Entwicklungsprozesses insgesamt übernahm. Infolge des Fehlens eines ausreichenden autonomen marktwirtschaftlichen Wachstums erhielt der repräsentativdemokratisch verfaßte Staat Aufgaben ähnlich denen der Volksdemokratien Osteuropas. Die Funktionserweiterung des Staates drückte sich institutionell sowohl in der Schaffung neuer Ministerien aus, wie etwa des Landwirtschaftsministeriums durch Dekret Nr. 3524 vom 1. August 1939 und des Wirtschafts- und Handelsministeriums durch Gesetz Nr. 7200 vom 8. Juli 1942, als auch in der Einrichtung staatlicher und halbstaatlicher Unternehmungen wie u. a. der Corporación de Fomento de la Producción (CORFO) im Jahre 1939, der Corporación de la Vivienda (CORVI) im Jahre 1953, der Empresa Nacional de Minería (ENAMI) und des Banco del Estado im Jahre 1960, sämtlich Institutionen, die die ökonomische und soziale Entwicklung des Landes fördern sollten ${ }^{53}$. Die erhöhten Aufgaben staatlicher Wirtschaftspolitik und -planung bedingten erweiterte Kompetenzen für die Exekutive, wenn sie den Entwicklungsprozeß erfolgreich steuern wollte. Die Notwendigkeit, dem Präsidenten auf Kosten der Rechte des Parlaments das geeignete Instrumentarium zur Steuerung des Wirtschafts- und Entwicklungsprozesses an die Hand zu geben, wurde vom Kongreß in zwei Verfassungsreformen anerkannt.

Unter dem Präsidenten Juan Antonio Rios trat am 23. November 1943 die erste dieser Reformen in Kraft, die einen wichtigen Schritt im Sinne einer Komplettierung der Verantwortung der Exekutive für die Wirtschafts- und Finanzpolitik des Landes bedeute. Der chilenische Kongreß verlor die Kompetenz, erhöhte Staatsausgaben zu beschließen. Auf seine Initiative hin waren in der Vergangen-

den Wahlen zum Abgeordnetenhaus und Senat. Im Jahre 1961 erzielten die Christdemokraten 16,0 Prozent, 1965 43,6 Prozent und 1969 31,0 Prozent der Stimmen. Sie erreichten damit im Abgeordnetenhaus 15,7, 55,8 respektive 37,3 Prozent der Mandate. In der Wahlperiode 1965-1969 verfügten sie somit über eine absolute Mehrheit der Mandate. In der Zusammensetzung des Senats, der alle vier Jahre halberneuert wird, steigerte sich ihr Anteil an Mandaten von 8,9 Prozent im Jahre 1961 auf 28,9 Prozent im Jahre 1965 und auf 44,0 Prozent im Jahre 1969. Bei den Wahlen von 1969, als ihr Stimmenanteil bereits wieder empfindlich rückläufig war, gewann die DC neun Senatorensitze hinzu. Als sie im Abgeordnetenhaus die absolute Mehrheit der Mandate einnahm, stellte sie im Senat kein Drittel der Mitglieder. So blockierte der Senat die Gesetzgebung der DC-Regierung.

53 CORFO ist die nationale Entwicklungsbehörde, CORVI die staatliche Institution zur Förderung des Wohnungsbaus, ENAMI die nationale Bergbaubehörde (ohne Kupfer). 1960 wurde auch der Departamento del Cobre gegründet, der später in die staatliche Kupferbehörde Corporación del Cobre (CODELCO) überging. Banco del Estado ist eine Staatsbank, nicht zu verwechseln mit der Banco Central, der Zentralbank, die bereits 1925 gegründet wurde. 
heit vielfach neue Posten in der Administration eingerichtet und die Löhne und Gehälter in der staatlichen Verwaltung aufgestockt worden. Die Schaffung öffentlicher Dienststellen, die Gewährung und Erhöhung von Löhnen, Gehältern und Gratifikationen der öffentlichen Verwaltung sowie der staatlichen und halbstaatlichen Unternehmen fiel nun ausschließlich dem Präsidenten zu (VfsArt. 45, Abs. 3). Noch verblieb dem Kongreß die Fähigkeit, durch Initiativen in Fragen von Löhnen und Gehältern im privaten Sektor sowie durch Steuer- und Sozialgesetze die Wirtschafts- und Finanzpolitik des Landes entscheidend zu beeinflussen. Hier setzte die Verfassungsreform vom 21. Januar 1970 an, die vom Präsidenten Eduardo Frei eingebracht wurde und die ausschließliche Kompetenz der Exekutive in den öffentlichen Ausgaben und Sozialausgaben sowie in der Festsetzung von Löhnen und Gehältern im privaten Sektor schuf.

Diese Verfassungsänderung, die die 1943er-Reform zu Ende führte ${ }^{54}$, war Teil eines weitgespannten Verfassungsreformprogramms der Christdemokraten, das vor dem Hintergrund der von dieser Partei geplanten und eingeleiteten Sozialrevolution verstanden werden muß. Der soziale und ökonomische Strukturwandel sollte sich legal, auf das Votum der Wählerschaft gestützt vollziehen, demokratisch induziert und dem Verdikt der Wählerschaft unterworfen werden. Deshalb legte Frei so viel Wert auf die Erneuerung der Verfassung selbst. Denn nur, indem das politische System mit den ihm eigenen Mitteln verändert würde, konnte sich der notwendige sozio-ökonomische Wandel legal einen Weg bahnen. Das streng legalistische Procedere, das Frei von vielen Kritikern vorgeworfen wird ${ }^{55}$, besaß sicherlich den Nachteil,' das Gelingen einer substantiellen Verfassungsreform in den für tiefgreifende Strukturveränderungen in Wirtschaft und Gesellschaft wichtigen Bereichen in Frage zu stellen. Tatsächlich verhinderte das Parlament denn auch die wenige Wochen nach Regierungsübernahme durch Frei im Kongreß eingebrachte Verfassungsreform ${ }^{56}$. Andererseits wurde durch das legale Vorgehen erreicht, daß die verabschiedeten Teilreformen in den Konsensus aller politischen Gruppen Chiles eingingen. Die Legalität der sozialen und ökonomischen Veränderung verschaffte dem durchgesetzten Wandel die erforderliche Legitimität. Der Gewinn, der darin liegt, wird allzuoft übersehen oder vorschnell der politischen Zielsetzung beschleunigten Wandels geopfert.

Die erste wichtige Verfassungsreform im Zuge der Frei'schen Sozialrevolution war die Änderung der Bestimmungen über das Eigentum, die der Kongreß mit den Stimmen der Christdemokraten, Kommunisten, Sozialisten und Radikalen am 16. Oktober 1966 annahm. In der Reform wurde die soziale Funktion des Eigentums hervorgehoben und seine Nutzung Beschränkungen und Verpflichtungen unterworfen, die sich aus dem Interesse der nationalen Gemeinschaft und einer Verbesserung der Lebensbedingungen aller Bürger herleiten. Der Staat erhielt das Recht zugesprochen, allein und ausschließlich die Naturressourcen und Produk-

54 S. dazu Eduardo Frei, La reforma constitucional en su contexto histórico-político, in: Frei u. a., Reforma (s. Anm. 9), S. 19-52. Der Beitrag stellt eine Ausarbeitung von Freis Rundf unk- und Fernsehrede vom 30. Dezember 1969 zur Verabschiedung der Verfassungsreform dar, die abgdr. ist in: El Mercurio, 31. 12. 1969 , S. 22.

55 S. unter anderem Klaus Esser, Durch freie Wahlen zum Sozialismus oder Chiles Weg aus der Armut, Reinbek bei Hamburg 1971, S. 104. Esser wechselt allerdings in Kriterien und Terminologie: so kann er praktisch in einem Atemzug den gesellschaftlichen "Transformationsprozeß unter der Regierung Frei“ als Vertiefung der "Krise der Demokratie" bezeichnen, in die alle Institutionen, "auch solche, die bis als Vertiefung der "Krise der Demokratie" bezeichnen, in die alle Institutionen, "auch solche, die bis Regierung rügen, der zum „Eckpfeiler der überkommenen Ordnung“ geworden sei (S. 103 f.).

56 Nach dem Reformenentwurf sollte der Präsident den Kongreß einmal während der Wahlperiode auflösen können und ihm zur Lösung eines Konfliktes zwischen Exekutive und Legislative das Referendum zur Verfügung gestellt werden, auch in der einfachen Gesetzgebung. Vgl. damit den Reformentwurf Allendes von 1971 weiter unten in der Abhandlung. 
tionsgüter zu übernehmen, die für die wirtschaftliche, soziale und kulturelle Entwicklung des Landes vorrangige Bedeutung haben ${ }^{57}$. Diese Verfassungsreform schuf die Grundlage für die Agrarreform, die nachfolgend mit Gesetz vom 28. Juli 1967 beschlossen wurde.

Die Verfassungsreform von $1970^{58}$ enthielt neben der schon genannten Ausweitung des Wahlrechts und der ausschließlichen Kompetenz des Präsidenten in der staatlichen Finanz- und Wirtschaftsplanung noch folgende Neuerungen: 1. Der Kongreß wurde ermächtigt, die Gesetzgebung in bestimmten Fragen, die Wirtschaft, Finanzen und Verwaltung betreffen, an den Präsidenten zu delegieren. Damit wurde mehr oder weniger der Verfassungspraxis entsprochen, von der Regelung durch Gesetzesdekrete aber ausdrücklich die Organisation des Kongresses und der richterlichen Gewalt sowie das Wahlsystem und die Grundrechte ausgeschlossen. 2. Die Gesetzgebung wurde an das Prinzip materieller Spezifizierung gebunden. Sie trat an die Stelle einer Gesetzgebung gemischten Gesetzgebungsinhalts, die den politischen Parteien gestattete, in die von der Regierung vorgelegten Gesetzentwürfe vielfältige, einzelne Personen oder Personengruppen begünstigende und teilweise im Widerspruch zur Intention des Gesetzes stehende Zusätze einzufügen (sog. leyes misceláneas). 3. Andere Änderungen betrafen die Rationalisierung der Gesetzgebung, etwa die Einrichtung von gemeinsamen Ausschüssen von Abgeordnetenhaus und Senat. In diesem Zusammenhang ist auch die Schaffung eines Verfassungsgerichts $\mathrm{zu}$ nennen, dem vor allem die Interpretation von Verfassung und Gesetzen anheimgestellt wird.

4. Die wichtigste Reform, die das Institutionssystem betraf, war die Einführung des Referendums in Fragen der Verfassungsreform, speziell im Konfliktfall zwischen Präsident und Kongreß. Die Wählerschaft erhielt somit die verfassunggebende Gewalt auch in Fragen der Weiterbildung des Verfassungsrechts, allerdings nur im Falle des Konflikts zwischen den Verfassungsorganen und auf Initiative des Präsidenten.

Wenn auch die Verfassungspläne der Christdemokraten nur teilweise realisiert werden konnten, so gelang ihnen doch die bedeutendste Verfassungsreform seit 1925. Die Stellung des Präsidenten im Verfassungssystem wurde verstärkt, ohne daß allerdings das Parlament entscheidend an Macht verlor. Die Reformen fügten sich in das bestehende politische System ein. Andere wirtschaftliche und gesellschaftliche Transformationen der Frei-Regierung bedurften keiner Verfassungsänderung, so etwa die Chilenisierung des Kupfers ${ }^{59}$, die Syndikalisierung der Landarbeiter, die Bildung von Nachbarschaftsverbänden ${ }^{60}$, Maßnahmen des Abbaus der außenwirtschaftlichen Abhängigkeit und der Organisation der randständischen Bevölkerung, die der Entwicklung des Landes dienen sollten ${ }^{61}$. Dieser insgesamt erfolgreich verlaufene Versuch einer "Revolution in Freiheit“ wurde nach sechs Jahren Frei-Regierung abrupt abgebrochen.

\footnotetext{
57 Vfsart. 10. S. die neue Ubersetzung der chilenischen Verfassung nach dem Stand von Dezember 1972 in der Beilage "Verfassungstexte“ zu VRU 1973, Heft 1.

58 S. dazu die" verschiedenen Beiträge $\mathrm{zu}$ den einzelnen Teilen und Aspekten der Verfassungsreform in Eduardo Frei u. a.: Reforma (s. Anm. 9).

$59 \mathrm{~S}$. dazu die Untersuchung von Karl-Heinz Stanzick, „El cobre es chileno“. Eine Untersuchung zur Nationalisierung des chilenischen Kupferbergbaus, in: Vierteljahresberichte, Probleme der Entwicklungsländer, 46/1971, S. 345-361.

60 Gesetz über die Sindicación campesina vom 29. 4. 1967, das den Landarbeitern das Recht einräumt, sich gewerkschaftlich zu organisieren, was vordem nur für die Industriearbeiter bestand; Gesetz über die Juntas de Vecinos vom 7. 8. 1968, das die Organisation der Siedler regelt und ihnen die rechtliche Handhabe für örtliche oder regionale Maßnahmen der „community“-Entwicklung auf Eigeninitiative verschafft.

61 Zur Konzeption und Durchführung der „Revolution in Freiheit ${ }^{\alpha}$, s. Dieter Nohlen, Chile (s. Anm. 19), S. $91-112$.
} 


\section{Das Verfassungsstatut von 1971}

Bei den chilenischen Präsidentschaftswahlen vom 4. September 1970 erreichte zum ersten Male in der Geschichte ein marxistischer Kandidat in freien Wahlen die Mehrheit der Wählerstimmen. Salvador Allende, Kandidat der Volkseinheit (Unidad Popular, UP'2 ), erhielt 36,5 Prozent der Wählerstimmen ${ }^{63}$. Da die chilenische Verfassung zur Wahl im ersten Wahlgang die absolute Mehrheit der abgegebenen gültigen Stimmen fordert, mußte der Kongreß die Wahl entscheiden. Im Kongreß besaß weder Allende noch der zweite Wahl, Alessandri, eine Mehrheit ${ }^{64}$. Die Christdemokraten mußten mit ihren Stimmen die Entscheidung herbeiführen. Seit 1925 hatte der Kongreß stets den Kandidaten gewählt, der im ersten Wahlgang die relative Mehrheit der Stimmen erhalten hatte ${ }^{65}$. Vor dem 4. September hatte eine heftige innenpolitische Debatte darüber stattgefunden, ob die Konvention des Kongresses, die relative Mehrheit des ersten Wahlgangs zu respektieren, inzwischen als Verfassungsnorm zu gelten habe. Diese These wurde von der politischen Rechten vertreten, die davon überzeugt war, die allgemeinen Wahlen zu gewinnen, im Kongreß aber die schwächste Position einnahm. Demgegenüber hatten die Christdemokraten und das Militär das Recht des Kongresses betont, zwischen den beiden stimmstärksten Kandidaten frei zu entscheiden.

Während die politische Rechte daran arbeitete, eine Regierungsübernahme Allendes $\mathrm{zu}$ verhindern ${ }^{66}$, richteten sich die Christdemokraten auf eine Unterstützung Allendes ein, die sie allerdings nicht bedingungslos gewähren wollten. Auch die Christdemokraten befürworteten einen beschleunigten und vertieften Strukturwandel, der sich allerdings innerhalb des bestehenden Institutionensystems und legal vollziehen müsse. Trotz wiederholter Erklärungen und Beteuerungen Allendes, daß eine UP-Regierung einen „eigenen chilenischen“, demokratischen und legalen Weg zum Sozialismus beschreiten werde, in welchem die bürgerlichen Freiheiten und der politische Pluralismus aufrechterhalten bleibe, lagen hier die größten Befürchtungen der DC. Für die Wahl Allendes im Kongreß mit ihren Stimmen forderte die DC deshalb ein Verfassungsstatut, in welchem die UP die Grundlagen der chilenischen Demokratie bekräftigen sollte. Das bestehende Verfassungsrecht sollte in diesem Sinne vertieft und erweitert werden.

Noch in der ersten Woche nach Allendes Wahlsieg trat eine fünfköpfige Kommission der DC zusammen, die auf der Basis von vier Kernforderungen, nämlich: Gewähr freier Wahlen, Autonomie der Universitäten, Informations- und Presse-

62 Die chilenische Volkseinheit UP setzte sich zusammen aus Kommunisten (PC), Sozialisten (PS), Radikalen (PR), der Unabhängigen Volksaktion (API), der Bewegung für die Volkseinheit (MAPU) und den Sozialdemokraten (PSD). Ihre Zusammensetzung hat sich im Laufe der Regierungszeit infolge einer Neugruppierung des Parteiensystems im Jahre 1971 verändert. S. dazu Dieter Nohlen, Chile, (s. Anm. 19), Kapitel IV, 2.5.

63 Jorge Alessandri Rodriguez bekam 34,9 Prozent, Radomiro Tomic 28,4 Prozent der abgegebenen gültigen Stimmen. Zur Interpretation der Wahlen von 1970 s. Juan Garcés, La pugna por la presidencia en Chile, Santiago 1971, und Dieter Nohlen, Chile (s. Anm. 19), Kapitel III.

64 Allendes UP-Parteien stellten 82 Kongreßmitglieder, Alessandri konnte mit den Stimmen der Nationalpartei und der Radikaldemokraten (zusammen 43), rechnen. Das Kontingent der Christdemokraten umfaßte 75 Kongreßmitglieder.

65 Von den 10 Präsidentschaftswahlen seit 1925 hatte der Kongreß 3 entschieden. Vor 1925 war es des öfteren vorgekommen, daß ein Kandidat, der nach Wählerstimmen gesiegt hatte, bereits keine Mehrheit der Wahlmänner auf sich vereinigte (vor 1925 wurde der Präsident indirekt gewählt) und folglich der in der Volkswahl unterlegene Kandidat entweder vom Wahlmännergremium oder vom Kongreß zum in der Volkswahl unterlegene Kandidat entw

66 Zunächst präsentierte die Rechte Alessandri als Gegenkandidat zu Allende für die Wahl im Kongreß unter der Formel, daß Alessandri nach seiner Wahl zurücktreten würde, um Neuwahlen zu ermöglichen. Rechtsextremisten planten einen Putsch gegen Allende, wie dann im Attentat auf General Schneider deutlich wurde, das die Wahl Allendes verhindern sollte. Wie sehr die Ubberlegungen einer nicht demokratischen Lösung von ausländischer, vor allem nordamerikanischer Seite geteilt wurden, geht aus den im Frühjahr 1972 veröffentlichen Dokumenten der amerikanischen Telefongesellschaft ITT hervor: Secretaría General de Gobierno, Los documentos secretes de la ITT, Santiago 1972. 
freiheit und unabhängige Stellung der Streitkräfte, ein in zwei Teile zerfallendes Dokument ausarbeitete. Teil eines des Dokuments ${ }^{67}$ enthielt acht Forderungen: 1. Aufrechterhaltung der Demokratie und ihrer Grundwerte, 2. Aufrechterhaltung der individuellen Freiheitsrechte, 3. Autonomie der Universitäten und Streitkräfte, 4. Aufrechterhaltung eines freien Schulwesens ohne Bevormundung und ohne offizielle politische Orientierung, 5. Freiheit der Meinungsäußerung in den Kommunikationsmedien, 6. Aufrechterhaltung unabhängiger Gewerkschaften und freier gesellschaftlicher Vereinigungen, 7. Respektierung der politischen Parteien als Ausdruck der Meinungsströmungen, in die die Bürgerschaft untergegliedert ist, und zusammengefaßt 8. Weiterbestehen einer pluralistischen Gesellschaft im politischen, sozialen und kulturellen Bereich in Chile. Teil zwei des Dokuments, der zunächst geheim blieb ${ }^{68}$, konkretisierte, wie die geforderten Garantien im einzelnen nach Form und Inhalt in die Verfassung aufgenommen werden könnten.

Die Antwort Allendes betonte die „Notwendigkeit, die volle Aufrechterhaltung eines Systems demokratischen Zusammenlebens und bürgerlicher Freiheiten zu garantieren " und verwies in den einzeln angesprochenen Forderungen der DC darauf, daß sie entweder in der Verfassung bereits verankert oder Inhalt des UP-Programms seien oder einer Haltung entsprächen, die er selbst in seinem politischen Wirken stets vertreten habe. Er ließ aber keinen Zweifel daran, daß das gegenwärtige System seinen Demokratievorstellungen nicht adäquat sei. Die Volksregierung habe sich eine doppelte Aufgabe gestellt: „Einerseits, die demokratischen Rechte und die Errungenschaften der Werktätigen aufrechtzuerhalten, zu vertiefen und effektiver $\mathrm{zu}$ machen; andererseits, die gegenwärtigen Institutionen umzuwandeln, um einen neuen Staat zu errichten, mit einem neuen Wirtschaftssystem, in welchem das Volk die wirkliche Macht ausübt ${ }^{69 " .}$. Obgleich die Antwort Allendes freundlich abgefaßt war und die Gemeinsamkeiten zwischen DC und UP hervorhob, lehnten die Christdemokraten sie ab, da sie „keine umfassende und zufriedenstellende Erwiderung auf die konkreten Vorschläge (sei), die die Christliche Demokratie vorgebracht hat, um ihre Vorstellungen in reale und effektive Garantien zu verwandeln"70. Die DC forderte eine Verfassungsreform in der kürzestmöglichen Frist, die Allende ihr schließlich „für die übereinstimmenden Ideen und Vorschläge, die die zwischen der Christlich-Demokratischen Partei und Salvador

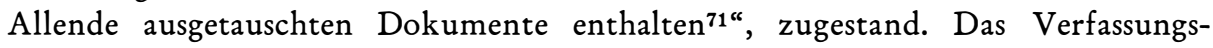
statut sollte mit den Stimmen beider politischen Gruppierungen im Kongreß verabschiedet werden. Daraufhin setzte sich in der einen Tag nach dieser Verlautbarung Allendes zusammengekommenen Junta Nacional der DC eine Mehrheit der Delegierten für die Wahl Allendes im Kongreß mit den Stimmen der DC ein ${ }^{72}$.

Die erste Verfassungsänderung unter der Regierung Allende vom 9. Januar 1971 (Gesetz Nr. 17.398) bekräftigte und spezifizierte die rechtsstaatlichen und bürgerlich-freiheitlichen Prinzipien einer pluralistischen Demokratie. Der Grundrechtskatalog wurde von einer mehr liberal-paternalistischen in eine mehr sozialstaatlichdemokratische Orientierung verwandelt: eingefügt oder in ihrem Gehalt erwei-

\footnotetext{
67 S. Mercurio, 24. September 1970. Das Schreiben wurde wiederabgdr. in Silva Sanchez, Primeras reformas constitucionales del Presidente Allende, Valparaiso 1971, S. 7-11.

68 S. Mercurio, 3. Oktober 1970, wiederabgedruckt in Silva Sanchez, Primeras reformas (s. Anm. 67), S. $11-13$.

69 Allende, zit. nach Silva Sanchez, Primeras reformas (s. Anm. 67), S. 19.

70 DC-Erklärung vom 30. September 1970, zit. nach El Mercurio vom 1. 10. 1970.

71 Allende-Erklärung, zit. nach La Nación, 3. 10. 1970.

72 S. zu dieser Phase auf dem Wege Allendes zur Ưbernahme der Regierung den Bericht von Dieter Nohlen, Die chilenische Christdemokratie nach den "historischen ${ }^{\alpha}$ Wahlen, in: Civitas, 10/1971, S. 232-262, hier besonders S. $251-258$.
} 
tert wurden unter anderem das Recht auf Arbeit, auf Beteiligung an den produzierten Gütern, das Streikrecht und das Recht, Gruppen zu organisieren. Allerdings ist das Statut rechtstechnisch wenig ausgereift. Da man nur einige Artikel revidierte, der Grundrechtsteii der Verfassung seit 1925 unverändert geblieben war und diese Fassung bereits fast wörtlich auf die teilweise im Verfassungstext verstreut befindlichen Grundrechte der Verfassung von 1833 zurückging, ist die mangelnde Kohärenz kaum verwunderlich. $\mathrm{Zu}$ einer Neuredaktion des gesamten Grundrechtskatalogs fehlte indes die Zeit ${ }^{73}$.

\section{Verfassungsreformziel: sozialistische Demokratie}

Die Vereinbarung eines Verfassungsstatuts zwischen Allende und den Christdemokraten kann nicht darüber hinwegtäuschen, daß es die Absicht der marxistischen Parteien und ihrer Regierung ist, die politische Verfassung Chiles im Sinne einer sozialistischen Demokratie umzugestalten. Wie wenig präzise umrissen die angestrebte sozialistische Demokratie in der Theorie auch ist und wie wenig einheitlich die gesellschaftspolitischen Zielvorstellungen der verschiedenen marxistischen Gruppen auch sind, so sticht doch der grundsätzliche Unterschied der Reformpläne Allendes zu den bisherigen Reformabsichten und Initiativen seit 1925 hervor. Den Vorgängern Allendes im Präsidentenamt ging es im wesentlichen um eine effizientere Struktur des Institutionensystems und um moderne Verfahrensweisen der politischen Willensbildung und Gesetzgebung. Allende dagegen plant, zugleich mit dem Übergang von der kapitalistischen zur sozialistischen Gesellschaft, den Wandel der repräsentativ-demokratischen Verfassung in eine Verfassung sozialistischen Typs. Allerdings hat Allende sich bei der Definition eines „eigenen chilenischen Weges zum Sozialismus" darauf festgelegt, die Reform der Verfassung mit den Mitteln des bestehenden Institutionensystems durchzusetzen. Er hat die Realisierung seiner Verfassungspläne von der Zustimmung des Parlaments und dem Konsensus der Wählerschaft abhängig gemacht. Wenigstens ist dies die Kernaussage des „eigenen chilenischen“, nämlich legalen und demokratischen Weges zum Sozialismus ${ }^{74}$. Soweit sich die marxistischen Gruppen in der Praxis an diese Methode halten, wird man sagen können, daß tatsächlich der Versuch gemacht wurde, innerhalb der Spielregeln der repräsentativen Demokratie ein grundlegend anderes Gesellschafts- und Demokratiemodell zu verwirklichen.

Die marxistischen Gruppen haben aber von Anbeginn der Regierung Allende die Möglichkeit eines demokratischen Weges zum Sozialismus sehr skeptisch beurteilt und statt eines schrittweisen Übergangs, der die realen Chancen im Auge behält, eine Mehrheit im Parlament oder in der Wählerschaft für ihre strukturverändernden Maßnahmen zu erhalten, den möglichst raschen Ausbau der Macht angestrebt ${ }^{75}$. Durch die Veränderungen im ökonomischen und sozialen Bereich (Verstaatlichungen, Agrarreform, Umverteilung) glaubten sie, die Voraussetzungen zu schaffen, die die sozio-ökonomische Basis ihrer politischen Gegner zerstören würde und - entsprechend der marxistischen Theorie - auch den politisch-institu-

\footnotetext{
73 Der Text des Verfassungsstatuts nebst erläuternden Bemerkungen ist abgdr. in Silva Sanchez, Primeras reformas (s. Anm. 67), S. 21-35. Für eine deutsche Ubersetzung der Verfassungsgarantien s. die Bei-

74 S. dazu Salvador Allende, Nuestro camino al socialismo - la vía chilena, Santiago 1971. Die Ambivalenz mancher theoretischer Position der chilenischen Marxisten wird deutlich in Régis Debray/Salvador Allende, Der chilenische Weg, Neuwied, Darmstadt, Berlin 1972.

75 Dazu im einzelnen Dieter Nohlen, Chile (s. Anm. 19), Kapitel IV.
} 
tionellen Ưberbau nachfolgend verändern müsse. Die Marxisten argumentierten aber auch, daß die bestehenden politischen Institutionen ein entscheidender Hemmschuh für die erfolgreiche Durchführung der sozialistischen Revolution seien, vor allem aber eine Gefahr für den Erhalt der Regierungsgewalt bedeuteten ${ }^{76}$. In dieser Perspektive räumten sie der Verfassungsfrage sogar Vorrang gegenüber der Transformation der sozio-ökonomischen Basis ein. Damit gewann die Verfassungsreform für die Marxisten wie für die Opposition zur Allende-Regierung einen gänzlich neuen Inhalt und Stellenwert. Die UP versteht unter der Verfassungsreform ein Mittel, ihre Macht zu verfestigen, da eine wirklich sozialistische Umwandlung der Verfassung für sie gleichbedeutend ist mit der Konstituierung der Arbeiterklasse als politisch dominante Klasse. Unter den klassenkämpferischen $\mathrm{Be}-$ dingungen, die die UP dem politischen Prozeß in Chile oktroyierte, war die an der repräsentativen Demokratie orientierte Opposition aus Christdemokraten und Nationalen zu keinen Konzessionen in Fragen der Verfassungsreform bereit, die das Institutionensystem und die bürgerlichen Freiheiten betrafen ${ }^{77}$. Vielmehr fühlte sich vor allem die Christdemokratie aufgerufen, die Verteidigung des institutionellen Erbes Chiles zu übernehmen.

Mit den Stimmen aller Parteien des Parlaments wurde am 11. Juli 1971 Verfassungsreform und Gesetz zur Nationalisierung der chilenischen Kupfererzvorkommen verabschiedet ${ }^{78}$. Alle Bodenschätze und Naturressourcen des Landes wurden unter die „absolute, ausschließliche, unveräußerliche und unverjährbare Verfügungsgewalt

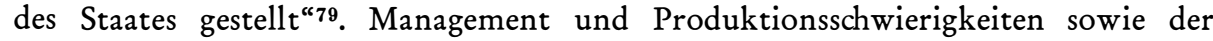
Streit mit den enteigneten US-Unternehmen um eine von den Nordamerikanern geforderte angemessene Entschädigung haben die chilenische Kupferindustrie, die 76 Prozent der chilenischen Außenhandelserlöse einbringt, in eine schwere Krise gestürzt ${ }^{80}$.

Andere Initiativen und Maßnahmen der Transformation von Wirtschaft und Gesellschaft fußten entweder auf der von der DC geschaffenen gesetzlichen Grundlage oder auf der extensiven Auslegung einer teilweise in Vergessenheit geratenen Gesetzgebung aus der Zeit der kurzlebigen Sozialistischen Republik Chile zu Beginn der dreißiger Jahre ${ }^{81}$, oder erfolgten im Vorgriff auf gesetzliche Grundlagen, die die UP gegenwärtig in Anbetracht der Mehrheitsverhältnisse im Kongreß nicht im Parlament einbrachte. Auf der Basis des Agrarreformgesetzes von 1967 schloß die UP-Administration bis Jahresende 1972 die Enteignung der Latifundien ab. Der Prozeß verlief jedoch vielfach illegal aufgrund revolutionärer Aktionen, für die der MIR (Bewegung der revolutionären Linken) verantwortlich zeichnete. Als Folge der Verunsicherung auch jener Eigentümer, die nach dem Gesetz nicht enteignet werden dürfen, und der rapide beschleunigten Reform, mit der die technischen und kreditiven Maßnahmen nicht Schritt hielten, blieb die Produktion der Agrarwirtschaft hinter den Erwartungen und Notwendigkeiten zurück. Ungelöst ist auch das Organisationsproblem der Landwirtschaft ${ }^{82}$. Die Verstaatlichung von Unternehmen und Banken geschah teilweise auf der Grundlage alter Dekrete,

76 Documenta socialista vom März 1972, abgdr. in: El Mercurio, 12./13. 3. 1972.

77 Dieter Nohlen, Chile (s. Anm, 19), S. $308 \mathrm{ff}$

78 S. dazu Karl-Heinz Stanzick, „El cobre es chileno ${ }^{\alpha}$ (s. Anm. 59), S. 354-356.

79 VfsArt. 10 nach der Reform vom 15. Juli 1971, zit. nach Sanchez Silva, Primeras reformas (s. Anm. 67), S. 44.

80 S. dazu im einzelnen Dieter Nohlen, Chile (s. Anm. 19), Kapitel IV, 1.4.

81 Ưber Inhalt, Rechtsnatur und heutige Bedeutung der unter der Sozialistischen Republik erlassenen Dekrete s. Eduardo Novoa Monreal, Vías legales para avanzar hacia el socialismo, in: Mensaje, 197/1971, S. 84-90. Zur Episode der Sozialistischen Republik s. jetzt die detaillierte Darstellung von Carlos Charlin, Del avion rojo a la República Socialista, Santiago 1972.

82 Zur Entwicklung der Landwirtschaft unter der UP s. Dieter Nohlen, Chile (s. Anm. 19), S. 179-186. 
teilweise ohne gesetzliche Grundlage. Als die Christdemokraten einen Entwurf zur Regelung der Verstaatlichung von Industrieunternehmen und Banken im Kongreß einbrachten, um die Maßnahmen der UP an Recht und Gesetz zu binden, kam es zum Verfassungskonflikt zwischen UP und DC, da der Präsident auf seinem Veto ebenso beharrte wie der Kongreß auf der von ihm verabschiedeten Verfassungsreform, die die bisherigen Verstaatlichungen teilweise als illegal bezeichnete ${ }^{83}$. Darüber hinaus ist auch im industriellen Sektor die Frage der Unternehmensverfassung ungelöst. Der staatskapitalistischen Tendenz der UP, die trotz aller gegenteiligen Verlautbarungen in der Praxis vorherrscht ${ }^{84}$, setzen die Christdemokraten ihr Modell von Arbeitunternehmungen (Empresas de Trabajadores) gegenüber. Abweichend von der entwicklungspolitischen Zielsetzung der Verstaatlichungspolitik im Industriesektor, die verstaatlichten Unternehmen $\mathrm{zu}$ Anführern wirtschaftlicher Effizienz und der nationalen Produktion zu machen, erwirtschaften sie erhebliche Defizite ${ }^{85}$.

Die Umstrukturierungsmaßnahmen mit ihren in aller Regel für die politische Basis der UP nicht günstigen Folgen führten die marxistische Diskussion immer wieder auf die zentrale Frage einer Reform des Institutionensystems zurück, so wie sie das UP-Programm durchzuführen versprach. Ihm zufolge sollte das Einkammersystem eingerichtet werden, die Gewählten sollten durch ihre Wähler überwacht und abgerufen werden können (recall-system), und alle Wahlen sollten $\mathrm{zu}$ gleicher Zeit stattfinden, um die notwendige Abstimmung zwischen den Staatsorganen herzustellen. Das UP-Programm bezeichnete die Volksversammlung als „höchstes Organ der Macht" und wies ihr auch die Aufgabe zu, über die Zuständigkeit und die Zusammensetzung des höchsten Gerichts zu entscheiden, welches eine neue Organisation der Justiz und Justizverwaltung einrichten soll. Der Justiz wurde die Aufgabe gestellt, „die Schichten, die die Mehrheit der Be-

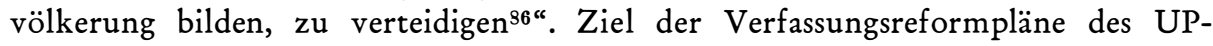
Programms war somit eine Vereinheitlichung der politischen Macht, die Aufhebung der Gewaltenteilung und die Bindung von Exekutive, Legislative und Justiz an den Willen der Mehrheit des Volkes „an sich“, dessen Vertretung die marxistischen Parteien für sich beanspruchen.

Trotz vielfacher Ankündigung legte Allende zunächst keinen Gesetzentwurf zur Reform der Verfassung vor. Da er immer wieder davon sprach, die Reform in einem günstigen Moment durchzuführen, wird man annehmen dürfen, daß er über lange Zeit glaubte, daß der opportune Zeitpunkt noch nicht gekommen sei. Selbst als die Kommunalwahlen vom April 1971 den UP-Parteien eine knappe Mehrheit der Stimmen einbrachten, zögerte Allende weiter mit der Vorlage. Offensichtlich verunsicherte gerade dieses Wahlergebnis Allende darin, eine Auseinandersetzung mit dem Kongreß um die geplante Verfassungsreform zu seinen Gunsten entscheiden zu können. Statt dessen wurden die strukturverändernden Maßnahmen in der Wirtschaft weiter forciert, wohl in der Annahme, die ökonomische Basis seiner innenpolitischen Gegner weiter zu schwächen und die Erosion des bestehenden Institutionensystems weiter voranzutreiben. In diesem Zusammen-

$83 \mathrm{Zu}$ den Differenzen in der Interpretation der Verfassung, die zum Verfassungskonflikt zwischen UPRegierung und Opposition führten, s. Dieter Nohlen (s. Anm. 19), S. 258-262.

84 S. etwa Salvador Allende, Nuestro camino (s. Anm. 74), S. 19. Vgl. dazu Boris Goldenberg, Chiles Weg zum Sozialismus, in: Aus Politik und Zeitgeschichte, 48/1971, S. 42.

85 Erste Untersuchungen haben dies bereits zu Jahresbeginn 1972 festgestellt. S. Teresa Jeanneret R., Area de propiedad social y captación de excedentes, in: Comentarios (s. Anm. 18), S. 139-154.

86 Das Programm der UP findet sich in deutscher Ubersetzung abgedr. in: A. Acquaviva, G. Fournial, P. Gilhodes, J. Marcelin, Das Chile der Volkseinheit, Frankfurt 1972 (= Marxistische Taschenbücher), S. 135-154, die Zitate auf S. 138 f. 
hang muß auch der Kampf um die Verstaatlichung der Papierindustrie (Presse) und an den Universitäten um die Vorherrschaft in den Universitätsgremien gesehen werden (zwei von drei Fernsehsendern werden von den Universitäten betrieben). Die UP-Parteien forderten indes immer wieder die Durchführung der Verfassungsreform, um die institutionellen Hemmnisse der sozialistischen Revolution aufzuheben.

Doch je länger Allende zögerte, das Reformprojekt einzubringen, desto geringer wurden seine Aussichten, eine Mehrheit des Parlaments oder, im Falle eines Konflikts mit dem Kongreß, eine Mehrheit der Wählerschaft für die Reform der Verfassung zu gewinnen. Inzwischen war zum einen die christdemokratische Opposition nicht mehr bereit, selbst Vorschläge, die in ihrem Programm standen, mit der UP zu verhandeln, da sie befürchtete, diese Reformen könnten die von der UP grundsätzlich in Frage gestellte Institutionalität Chiles weiter aushöhlen und die Macht der UP verfestigen. Zum anderen zeigten sich die gravierenden wirtschaftlichen Folgen einer Politik, die auf eine kurzfristige Zielsetzung, die Verbreiterung der politischen Basis der UP, angelegt war und die die bei einem möglichen Scheitern dieser Strategie auftretenden politischen Gegenwirkungen nicht berücksichtigte.

Erst im November 1971 legte Allende schließlich dem Kongreß einen Gesetzentwurf vor, der nur Teile seines Reformvorhabens enthielt und in der Terminologie jegliche Anspielung auf die Einrichtung einer sozialistischen Demokratie vermied. Das Zweikammersystem sollte durch eine einzige Kammer ersetzt und das Parlament am gleichen Tag wie der Präsident gewählt werden. Die zentrale Verfassungsfigur blieb der Präsident. Er sollte das Recht erhalten, das Parlament einmal während der Wahlperiode auflösen zu können. Es sollte das Volksbegehren eingeführt werden, das durch Aufbringung von 5000 Unterschriften oder durch Initiative der CUT eingeleitet werden kann ${ }^{87}$.

Der rechts-unabhängige El Mercurio sah in dem Reformprojekt „einen der schlimmsten Schläge, die unser Institutionensystem erfahren kann" und erklärte das Einkammersystem „zu einem weiteren Instrument der sozialistisch-marxistischen Revolution", die es abzuwenden gelte ${ }^{88}$. Auch die Christdemokraten stellten zum Reformvorhaben, das seinem Ziel nach von ihnen stammen könnte, fest, „daß in dem gegenwärtigen politischen Kontext das Einkammersystem ein neuer Schritt der bekannten marxistischen Taktik sei, um jeden Preis die totale Macht zu gewinnen" und lehnten es deshalb ab ${ }^{89}$. Bei der ersten Abstimmung in der Parlamentskommission für Verfassung, Recht und Justiz stimmten nur vier Kommissionsmitglieder für die Reform, acht dagegen, einer enthielt sich der Stimme ${ }^{90}$. Mit den Oppositionsstimmen versahen die Christdemokraten das Projekt mit einer wesentlichen Änderung: Die Auflösung des Parlaments soll von einem Referendum abhängig gemacht werden. Verliert der Präsident das Referendum, so hat er zurückzutreten und Neuwahlen für die Bestellung seines Nachfolgers auszuschreiben.

Der Gegenentwurf der Opposition macht deutlich, daß sich die politische Verfassung unter der UP-Regierung vom Spielregelsystem der politischen Ausein-

87 Die CUT, Central Unica de Trabajadores, ist die Einheitsgewerkschaft der chilenischen Industriearbeiter. Der Text des Verfassungsreformentwurfs ist abgdr. in: La Nación, 12. 11. 1971.

88 El Mercurio, 12. 11. 1971.

89 Erklärung der DC vom 22. 11. 1971, zit. nach El Mercurio, 23. 11. 1971

$90 \mathrm{La}$ Prensa, 26. 11. 1971. 
andersetzung zum Instrument des Kampfes um die politische Macht entwickelt hat. Während die Regierung versucht, die bürgerlichen Institutionen, die von den Marxisten als Instrumente der bürgerlichen Klassenherrschaft bezeichnet werden, $\mathrm{zu}$ überwinden, weil sie der Weg und die Mittel seien, der Bourgeoisie wieder an die Macht zu verhelfen ${ }^{91}$, will die Opposition die Gegengewichte zum Präsidenten im bestehenden Verfassungssystem ausbauen, um eventuell sogar Allende aus der Macht zu verdrängen. Der sozio-ökonomische Wandel, von der UP als Übergang (oder als Übergang zum Übergang) von der kapitalistischen zur sozialistischen Gesellschaft verstanden ${ }^{92}$ und in den Kategorien einer klassenkämpferischen Auseinandersetzung vorangetrieben, hat die Verfassungsfrage zu einer ideologischen und gesellschaftlichen Machtfrage erhoben. Das chilenische Militär, auf die Verteidigung des gültigen Rechts eingeschworen ${ }^{93}$, seinem Wandel aber nicht verschlossen ${ }^{94}$, sichert gegenwärtig, daß diese Auseinandersetzung weiterhin friedlich verläuft und die bewaffnete Auseinandersetzung, die in der Taktik der linken und rechten Extremisten liegt, unterbleibt. Die Parlamentswahlen vom 4. März 1973 werden einen ersten Hinweis darauf geben, ob die sozio-ökonomische Transformation innerhalb der pluralistisch-demokratischen Traditionen der chilenischen Politik weitergeführt wird oder ob sich ein neues Demokratieverständnis, die Diktatur des Proletariats, mehrheitlich Bahn brechen kann ${ }^{95}$.

91 Diese Ansicht teilen der linke Flügel der Sozialisten unter Carlos Altamirano und der MIR. S. Documenta socialista (Anm. 76) und La izquierda hace su balance. Segundo encuentro del movimiento Cristianos por el Socialismo, 24.-26. 11. 1972, in: Punto Final 172/1972, Beilage S. 21-48.

92 Auf die ideologischen Differenzen unter den Marxisten in der Frage, ob sich Chile unter der UP-Regierung im Ubergang zum Sozialismus befinde oder ob nur die Voraussetzungen für den eigentlichen UUbergang (nach der absoluten Machtübernahme der Marxisten) geschaffen würden, sei hier nur verwiesen. Für eine nähere Untersuchung s. Dieter Nohlen, Chile (s. Anm. 19), S. 289-308.

93 S. La doctrina del General, in: Ercilla, 1822/1970, S. 11 .

$94 \mathrm{~S}$. das Interview des im Oktober 1972 zum Innenminister berufenen ranghöchsten Militärs, General Carlos Prats Gonzales, in: Ercilla 1950/1972, S. 7-13.

95 Zum Ergebnis der für den weiteren Verlauf der dhilenischen innenpolitischen Entwicklung wichtigen Wahlen s. Dieter Nohlen, Chile (s. Anm. 19), S. 329-338. 
inherited an anglo-based legal system should or perhaps, should not, be tackled. Some of the present day problems in regard to land policy arose out of the strains and tensions imposed by the indigenous customs in regard to succession to land and the practice of a shifting agriculture system; these problems were aggravated by the colonial administration and the allocation of land to European settlers. The British Colonial Office set up a Commission in 1953 to review the land problems and recommend reforms. These reforms were accepted and put into effect by the Kenya Government from 1959-1963. They gave rise to some problems and the position was again reviewed in 1966 when further reforms were recommended. The reforms are based on the adjudication and consolidation of land titles coupled to a system of registration. An attempt has been made to apply the reforms to land held under customary tenure as well as that held under the colonial system of law and although problems have arisen, the Kenya Government is determined to tackle these in a progressive manner.

\section{Socio-Economic Change and Constitutional Reform in Chile, 1925-1972}

\section{By Dieter Nohlen}

Since $1810 / 1817$, the exceptionally stable system of constitutional government in Chile has produced only two constitutions: those of 1833 and 1925, and a high degree of legitimacy. Traditional institutions were democracized along Western lines. They gradually integrated different social strata, allowing political parties to take roots in the social fabric as an element of political stability. The 19th century political parties remained dominant for about 100 years. In contrast to the political, i. e. institutional evolution, social and economic development lagged behind. The Popular Front presidency in the late thirties, however, put new accents on industrialization and social change such as increase in population and urbanization. When private sectors could not cope with these problems the State seized control over economic development by promoting, financing and managing industrial activities. But the general situation even deteriorated, given the maintenance of oligarchical and obsolete structures. Hence, internal polarization and dependency as signs of underdevelopment increased (Chapt. I). Against this socio-economic background emerged the modern political parties (Christian-Democratic, Communist and Socialist). They initiated an overall mobilization and urged structural reforms in economy and society. Both the Christian-Democratic and Marxist programs, competing in the 1964 presidential election, called for reform of the 1925 constitution (Chapt. II). Within the framework of representative democracy Eduardo Frei initiated and partly achieved extensive reforms with the aim of modernizing economic, social and political institutions and procedures (Chapt. III). His successor, Salvador Allende, head of a coalition government with Marxist parties in dominant position, sought to fundamentally change the political system into a socialist society. Christian Democrats demanded constitutional guarantees concerning civil liberties and pluralistic democracy. Allende had to agree in order to get Christian-Democratic votes necessary to obtain the absolute majority during the second election round in Congress (Chapt. IV). Allende obtained the constitutional change allowing the nationalization of mines and foreign companies exploiting copper by unanimous vote. His attempts gradually to create a socialist system, 
however, faced resistance within the opposition parties dominating Congress. They object to direct and illegal revolutionary actions and the erosion of the democratic institutions. Thus the constitutional issue is really a conflict between the Marxist and Western ideologies (Chapt. V). 